\title{
Über die Trennung des Thoriums und der Ceriterden durch neutrales Natriumsulfit.
}

\author{
Von \\ Hermann Grossmann.
}

Über die Sulfite der seltenen Erden finden sich in der Literatur vereinzelte Angaben, die nur zum Teil zutreffend sind.

Von besonderem Interesse ist bei der Bedeutung des Thoriums für die Gasglühlichtindustrie eine neuere Arbeit von CHAvastelox ${ }^{1}$ nach welcher sich Thorium mit neutralem Natriumsulfit bequem von den dreiwertigen Ceriterden trennen lärst. Setzt man nämlich eine konzenzentrierte warme oder kalte Lösung von $\mathrm{Na}_{2} \mathrm{SO}_{3}$ zu Thoriumsalzlösungen, so löst sich der anfangs gebildete Niederschlag im Überschufs des Fällungsmittels wieder auf, während vorhandene Ceriterden und eine geringe Menge Thorium als unlösliche Sulfite ausfallen. Es fehlt jedoch völlig an quantitativen Versuchen über die Brauchbarkeit der Trennung bei geringem Thoriumgehalt, noch ist der Mechanismus der Reaktion zwischen Thoriumsalzen und Natriumsulfit aufgeklärt.

Bei Wiederholung der Versuche von Chavastelon wurde zuerst nochmals die Reaktion zwischen Natriumsulfit and reinem Thoriumsalz (es kam käufliches Nitrat zur Anwendung) studiert. Es zeigte sich bald, dals seine Angaben nur zum Teil richtig waren. Während bei Anwendung kalter konzentrierter Sulfitlösung der anfangs ausfallende gallertartige Niederschlag sich glatt auflöste, fielen beim Erhitzen der Lösung erhebliche Mengen amorpher Niederschläge verschiedener Zusammensetzung aus. Es erschien deshalb die Anwendung warmer Lösungen von vornherein nicht günstig für eine Trennung des Thoriums. Um die Frage der praktischen Brauch-

1 Compt. rend. $130,781$. 
barkeit der Trennung zu prufen, stellte ich Lösungen her, deren Gehalt an Thorium ungefähr dem Verhaltnis entsprach, in welchem dieses Element im Monazitsand enthalten ist. Vorher wurde jedoch das Verhalten einer neutralen Lösung der Ceriterden, wie man sie leicht ans dem sogenannten Cerium oxalicum purum des Handels herstellen kann, ${ }^{1}$ gegen $\mathrm{Na}_{2} \mathrm{SO}_{3}$. geprüft. Die Fällung verläuft quantitativ: Im Filtrat lassen sich charakteristische Absorptionsstreifen des Didyms nicht mehr erkennen. Die angewandte Lösung enthielt $16.744 \mathrm{~g}$ Oxyde in $100 \mathrm{ccm}$, während der Gehalt der Thoriumnitratlösung $1.754 \mathrm{~g} \mathrm{ThO}_{2}$ im gleichen Volum betrug. Dieses Gewichtsverhaltnis von 9.5:1 entspricht ungefähr dem Thoriumgehalt des Monazits.

Die gemischten Lösungen, je $10 \mathrm{ccm}$, wurden tropfenweis unter automatischer Ruhrung in stark überschüssige, kalte, konzentrierte Sulfitlösung $(20-50 \%)$ gegeben, das Umrthren 3-4 Stunden fortgesetzt, die Niederschläge abfiltriert und das Filtrat durch $\mathrm{HCl}$ zersetzt, bis zur Entfernung des Schwefeldioxyds erhitzt und mit Ammoniak gefällt. Es ergab sich jedoch, dafs bei Anwendung dieser Mengenverhältnisse, bei der die Thoriummenge, obwohl absolut genommen gering, doch gröfser war als in vielen technischen Produkten, nur geringe Mengen Thorium in Lơsung gingen (bis zu $10 \%$ ).

Aulserdem enthielt dieses Thorium noch geringe Mengen Cer, wie die braune Farbe des Oxyds bewies. 90\% Thorium und mehr blieben mit den Sulfiten der Cererden im Niederschlag und lassen sich leicht durch $\mathrm{H}_{2} \mathrm{O}_{2}$ nach der Methode von Lecoq De BorsBaddran ${ }^{2}$ nachweisen. Nach diesen unter den verschiedensten Verhältnissen (Variierung der Sulfitkonzentration und der Temperatur) ausgefuhrten erfolglosen Versuchen, besitzt die Trennungsmethode von CHAvastelon bei geringem Thoriumgehalt keinen praktischen Wert.

Es blieb dennoch noch die Frage zu lősen, auf welchen chemischen Vorgang die Reaktion zwischen Thoriumnitrat und Natriumsulfit beruhe, denn in dem Verhalten dieses Reagenzes liegt ein charakteristischer Unterschied des Thoriums und des Zirkoniums einerseits und der dreiwertigen Ceriterden.

Auf das Verhalten des Ammoninmsulfits gegen lösliche Zirkonsalze hatte 1844 bereits Hakmans ${ }^{*}$ aufmerksam gemacht. Nach ihm

1 Durch Kochen mit konz. $\mathrm{HNO}_{8}$ erhalt man die Nitrate, die Lösung wird vorsichtig mit $\mathrm{NH}_{\mathbf{3}}$ neutralisiert.

2 Compt. rend. 100, 605; J. 1885, 493.

"Journ. prakt. Chem. 38 (1884), 85. 
gibt dieses Reagenz einen gallertartigen Niederschlag, der sich im Überschul's des Fällungsmittels löst. Diese Lösung soll weder durch $\mathrm{NH}_{3}$ noch durch Allablihydroxyd tällbar sein. Diese Angabe ist jedoch nicht zutreffend. Die Lösung in überschüssigem $\left(\mathrm{NH}_{4}\right)_{8} \mathrm{SO}_{8}$ wie im neutralem Natriumsulfit, welches sich gleich verhält, wird durch $\mathrm{KOH}$ und $\mathrm{NH}_{3}$ sofort gefällt. Die klaren Lösungen in uberschüssigem Sulfit scheiden übrigens nach 12-24 Stunden in der K̇ulte, in der Hitze sofort, gallertartige, schwer filtrierbare Niederschlagge $a b$, die nach dem Trocknen auf Ton zu glasigen Massen werden, die sich selbst in konzentriertem $\mathrm{HCl}$ schwer lösen. Die Analyse eines in der Kalte aus Natriumsulfit erhaltenen nnd getrockneten Niederschlage ergab auf ein $\mathrm{Mol} . \mathrm{ZrO}_{2}(34.98 \%) \quad 1 / 2 \quad \mathrm{Mol} . \mathrm{Na}_{2} \mathrm{O}$ $(8.88 \%$ ). Die Bestimmung der schwefligen Säure gelang bisher weder durch Schmelzen mit Salpeter und Soda, noch auf anderem Wege. Wahrend $\left(\mathrm{NH}_{4}\right)_{2} \mathrm{SO}_{3}$ sich gegen Zirkonsalze gleich dem Natriumsalz verhalt, zeigen beide Verbindungen gegen Thoriumnitrat abweichendes Verhalten. ${ }^{1}\left(\mathrm{NH}_{4}\right)_{2} \mathrm{SO}_{3}$ fällt in verdünnter Lösung quantitativ das Thorium als basisches Sulfit und löst bei sehr starker Konzentration geringe Mengen des Niederschlages, während der durch Natriumsulfit gefällte Niederschlag sich uberaus leicht im Überschufs des Fạllungsmittels wieder löst.

Aus der konzentrierten kalten Lösung von $\mathrm{Th}\left(\mathrm{NO}_{3}\right)_{4}$, welche mit der zur Wiederauflösung des anfangs entstandenen, gallertartigen, basischen Sulfits gerade ausreichenden Menge Natriumsulfit versetzt war, schied sich nach $\mathrm{zwei}$ - bis dreitägigem Stehen über konzentriertes $\mathrm{H}_{8} \mathrm{SO}_{4}$ ein weifses Pulver ab, dessen Analysen auf ein basisches Thoriumnatriumsulfit von konstantem Molekularverhältnis beider Metalle, wie mehrere Darstellungen ergaben, wies. Die Lösung gibt mit Alkalien und Ammoniak Niederschläge von Thoriumhydroxyd, die Komplexităt der Doppelverbindung ist demnach keine sehr starke.

Thorium und Natrium wurden wie ablich bestimmt. Die Bestimmung der schweflichen Säure geschah durch Lösung des Doppelsulfits in Alkalikarbonat und Kochen der Lösung mit Brom. Nach vorsichtiger Neutralisation mit $\mathrm{HCl}$ und Fortkochen des Broms wurde erst $\mathrm{BaCl}_{3}$ gefallt. Die Oxydation verläuft nicht ganz quantitativ und die gefundenen Werte waren meist etwas zu niedrig, doch versagten die jodometrischen Methoden in diesem Falle durchaus und

1 Es wäre interessant zu untersuchen, ob auf diesem Wege eine Scheidung des Zirkons und Thoriums ausführbar sei. 
ergaben zu verschiedene Werte. Die Analyse des zuerst erhaltenen Produktes, welche auf Ton getrocknet war, führte zu der Formel:

$$
\mathrm{Th}_{2}(\mathrm{OH})_{2}\left(\mathrm{SO}_{3}\right)_{3} 2 \mathrm{Na}_{3} \mathrm{SO}_{3}+22 \mathrm{H}_{3} \mathrm{O} \text {. }
$$

$0.600 \mathrm{~g} \mathrm{~S}$ ergaben $0.2274 \mathrm{~g} \mathrm{ThO}_{2}$ und $0.1434 \mathrm{~g} \mathrm{Na}_{2} \mathrm{SO}_{4}$

$0.690 \mathrm{~g} \mathrm{~S}$ ergaben $0.2584 \mathrm{~g} \mathrm{ThO}_{2}$

$0.471 \mathrm{~g} \mathrm{~S}$ ergaben $0.1760 \mathrm{~g} \mathrm{ThO}_{\mathrm{g}}$ und $0.1100 \mathrm{~g} \mathrm{Na}_{2} \mathrm{SO}_{4}$

$0.432 \mathrm{~g} \mathrm{~S}$ ergaben $0.3604 \mathrm{~g} \mathrm{BaSO}_{4}$

$0.589 \mathrm{~g}$ S ergaben $0.4960 \mathrm{~g} \mathrm{BaSO}$,

$0.3684 \mathrm{~g} \mathrm{~S}$ ergaben $0.3210 \mathrm{~g} \mathrm{BaSO}_{4}$.

Ber. Th 33.53 ;

$\mathrm{Na} 6.65$;

S 11.57 .

Gef. Th 32.92, 32.85, 33.31; Na 7.75, 7.57; S 11.46, 11.97, 11.56.

Eine zweite Darstellung ergab bis auf den Wassergehalt, auf den ich keinen zu grofsen Wert lege, die Formel:

$$
\mathrm{Th}_{8}(\mathrm{OH})_{2}\left(\mathrm{SO}_{3}\right)_{8} 2 \mathrm{Na}_{2} \mathrm{SO}_{3}+12 \mathrm{H}_{2} \mathrm{O} \text {, }
$$

welche am besten den Analysenresultaten entspricht.

$0.430 \mathrm{~g} \mathrm{~S}$ ergaben $0.1930 \mathrm{~g} \mathrm{ThO}_{2}$ und $0.1080 \mathrm{~g} \mathrm{Na}_{2} \mathrm{SO}_{4}$

$0.506 \mathrm{~g} \mathrm{~S}$ ergaben $0.463 \mathrm{~g} \mathrm{BaSO}$.

Ber. Th $38.49 ; \mathrm{Na} \mathrm{7.63;} \mathrm{S} 13.27$.

Gef. Th 39.45 ; Na 8.16 ; S 12.59 .

Das basierte Thoriumnatriumsulfit ist in reinem Wasser nicht löslich, dagegen leicht in verdinnter $\mathrm{HCl}$. Von Alkalikarbonat und Bikarbonat wird es gleichfalls leicht und fast vollständig gelöst, jedoch traben sich diese Lösungen beim Kochen.

Neutrales Kaliumsulfit verhält sich dem Natriumsalz ähnlich. Der ausfallende Niederschlag von basischem Thoriumsulfit (der alkalihaltig ist) löst sich im Überschufs des Fällungsmittels wieder auf. Aus der klaren Lösung schied sich über konzentrierter $\mathrm{H}_{2} \mathrm{SO}_{4}$ nach 12 Stunden eine gallertartige Masse aus, die abgeprefst wurde und nach dem Trocknen ein amorphes wejfses Pulver darstellte. Die Analyse ergab die von der Zusammensetzung des Natriumsalzes abweichende Formel:

$$
\mathrm{Th}(\mathrm{OH})_{2} \mathrm{SO}_{8} 2 \mathrm{~K}_{2} \mathrm{SO}_{3}+10 \mathrm{H}_{2} \mathrm{O} \text {. }
$$

$0.684 \mathrm{~g} \mathrm{~S}$ ergaben $0.1830 \mathrm{~g} \mathrm{ThO}_{2}$ und $0.2780 \mathrm{~g} \mathrm{~K}_{2} \mathrm{SO}_{4}$

$0.560 \mathrm{~g} \mathrm{~S}$ ergaben $0.1480 \mathrm{~g} \mathrm{ThO}_{2}$ und $0.2920 \mathrm{~g} \mathrm{~K}_{2} \mathrm{SO}_{4}$

$0.4710 \mathrm{~g} \mathrm{~S}$ ergaben $0.3700 \mathrm{~g} \mathrm{BaSO}$,

$0.3750 \mathrm{~g} \mathrm{~S}$ ergaben $0.2904 \mathrm{~g} \mathrm{BaSO}_{4}$. 
Ber. Th 28.81; K 18.58; S 11.41 .

Gef. Th 29.25, 29.61; K 18.25, 18.18; S 10.78, 10.63 .

Versuche, durch Lösen von Thoriumhydroxyd in Bisulfitlösungen Doppelverbindungen zu erhalten, führten nicht zum Ziele. Lösungen von Thoriumsulfit werden durch Bisulfite gefällt. Die Niederschläge sind im Überschufs des Fällungsmittels nur wenig löslich. Neutrale Lösungen der Ceriterden werden ebenfals durch Alkalibisulfit gefällt, nur reines Cerosalz gibt leicht lösliche Niederschläge entsprechend der grofsen Löslichkeit des Cerosulfits in freier schwefliger Säure. ${ }^{1}$

Schweflige Säure fällt aus konzentrierten Lösungen von Thoriumnitrat einen starken weifsen Niederschlag von basischem Sulfit, im Filtrat bleibt infolge der Anwesenbeit der freien Salpetersäure etwas Thorium gelöst.

Die Analyse des amorphen Niederschlages, der auf Ton getrocknet war, ergab die Formel:

$$
\mathrm{Th}_{2}(\mathrm{OH})_{2}\left(\mathrm{SO}_{3}\right)_{3}+37 \mathrm{H}_{2} \mathrm{O} \text {. }
$$

$0.525 \mathrm{~g} \mathrm{~S}$ ergaben $0.2000 \mathrm{~g} \mathrm{ThO}_{2}$

0.387 g S ergaben $0.1416 \mathrm{~g} \mathrm{ThO}_{2}$

$0.329 \mathrm{~g} \mathrm{~S}$ ergaben $0.1210 \mathrm{~g} \mathrm{ThO}_{2}$

$0.2722 \mathrm{~g} \mathrm{~S}$ ergaben $0.1450 \mathrm{~g} \mathrm{BaSO}_{4}$.

Ber. Th $33.09 ; \quad$ S 6.85 .

Gef. Th $33.49,32.15,32.33 ; \mathrm{S} 7.27$.

Das neutrale Sulfit hat CLEvE ${ }^{2}$ durch Lösen von $\mathrm{ThO}_{2}$ in wässeriger $\mathrm{SO}_{2}$ erhalten. DavidsoHN ${ }^{3}$ erhielt an Stelle der Clueveschen Formel Th $\left(\mathrm{SO}_{3}\right)_{2} \mathrm{H}_{2} \mathrm{O}$ ein Salz von der Zusammensetzung $\mathrm{Th}\left(\mathrm{SO}_{3}\right)_{2} \cdot 4 \mathrm{H}_{2} \mathrm{O}$.

Thoriumhydroxyd löst sich nur sehr schwer in der Säure auf und fälllt beim Erwärmen der Lösung nieder. Dieses Verhalten zeigen auch die Sulfite des Cers, Lanthans und Didyms, ${ }^{4}$ von denen das Lanthansulfit sich beim Einleiten von $\mathrm{SO}_{2}$ in eine kalte wässerige Suspensension des Hydroxyds bildet und zwar leichter als das Thoriumsulfit, aber viel schwerer als Cersulfit in Lösung geht. Didymoxyd löst sich nach den Beobachtungen von Marianad ${ }^{5}$

1 Bull. 8oc. chim. [2] 21, 539.

2 Bull. soe. shim. [2] 21, 121.

${ }^{3}$ Inaugural-Dissertation, Berlin 1902, S. 36.

4 Cleve, Bull. soe. ohim. [2] 21, 251.

${ }^{5}$ Ann. chim. phys. [3] 38, $16 \overline{7}$. 
ebenfalls sehr leicht in wässeriger $\mathrm{SO}_{2}$ und fällt beim Erwärmen der Lösung nieder. Auf diesem verschiedenen Verhalten der Hydroxyde gegen schweflige Säure beruht das Patent der Herren Buddeus, Predssner, Itzic und Oppenheming, ${ }^{1}$ welche die Monazitoxyde mit schwefliger Säure behandeln, wobei ein relativ thorreiches Material zurückbleibt, während die Cerbasen meist in Lösung gehen.

Einige Beobachtungen über die Sulfite des Cers und Lanthans seien hier noch kurz angefügt. Lanthansulfit wurde beim Einleiten von $\mathrm{SO}_{2}$ in eine wăsserige Suspension von frisch gefalltem Lanthanhydroxyd unter Eiskühlnng erhalten. Die vom überschüssig nicht gelösten Hydroxyd abfiltrierte Flüssigkeit ergab sofort eine zuerst amorphe weifse Abscheidung, die nach kurzem Stehen über $\mathrm{H}_{2} \mathrm{SO}_{4}$ kristallinisch wurde und aus farblosen weifsen Nadeln bestand. Beim Kochen der Lösung fiel ein amorpher Niederschlag aus, der sich beim Abkühlen, falls noch freie Säure vorhanden war, wieder löste. Bei längerem Kochen fallen starke basische, in der Kälte nicht lösliche Niederschläge aus. Die Analyse ergab mit Ausnahme des wieder zu niedrig gefundenen S.Gehaltes auf die Formel $\mathrm{La}_{8}\left(\mathrm{SO}_{3}\right)_{3}+3 \mathrm{H}_{2} \mathrm{O}$ stimmende Zahlen

$$
\begin{aligned}
& 0.2478 \mathrm{~g} \mathrm{~S} \text { ergaben } 0.1412 \mathrm{~g} \mathrm{La}_{2} \mathrm{O}_{\mathrm{s}} \\
& 0.2166 \mathrm{~g} \cdot \mathrm{S} \text { ergaben } 0.1252 \mathrm{~g} \mathrm{La} \mathrm{O}_{3} \\
& 0.1830 \mathrm{~g} \mathrm{~S} \text { ergaben } 0.2214 \mathrm{~g} \mathrm{BaSO}_{4} \text {. }
\end{aligned}
$$

$$
\begin{array}{lr}
\text { Ber. La } 48.52 \text {; } & \text { S } 16.84 . \\
\text { Gef. La } 48.53,49.27 ; & \text { S } 16.20 .
\end{array}
$$

Die aus Lanthanchlorid durch $\mathrm{Na}_{2} \mathrm{SO}_{3}$ erhaltene kristallinische Fällung enthielt ca. $2 \%$ Natriumsulfit, eine Doppelverbindung liegt jedoch nicht ror.

Der durch Fällung aus Ceronitrat erhaltene Niederschlag, der schleimig war, ergab nach sorgfaltigem Auswaschen und Trocknen auf Ton eine rosa gefärbte Masse von der Forwel $\mathrm{Ce}_{2}\left(\mathrm{SO}_{3}\right)_{3}+7 \mathrm{H}_{2} \mathrm{O}$

$$
\begin{aligned}
& 0.431 \mathrm{~g} \mathrm{~S} \text { ergaben } 0.2305 \mathrm{~g} \mathrm{CeO}_{\mathrm{g}} \\
& 0.389 \mathrm{~g} \mathrm{~S} \text { ergaben } 0.1840 \mathrm{~g} \mathrm{CeO} \\
& 0.385 \mathrm{~g} \mathrm{~S} \text { ergaben } 0.3980 \mathrm{~g} \mathrm{BaSO}_{4}
\end{aligned}
$$

1 Chem. Centrbl. 1897 I, 486. 
Durch Lösen von Cerokarbonat in schwefliger Säure wurde ein in schwach rosa gefärbten Nadeln kristallisiertes Cerosulfit nach längerem Stehen über konzentrierter $\mathrm{H}_{2} \mathrm{SO}_{4}$ erhalten. Nur bei Anwendung konzentrierter Lösungen gelingt die Darstellung, aus verdünnten Lösungen wird schliefslich nur Cerosulfat erhalten. Die Lösung der Cerosulfits in schwefliger Säure zeigt besonders schön die Fällung in der Hitze und Lösung beim Abkïhlen. Ich möchte diese in Reagenzglas leicht ausführbare Reaktion als Vorlesungsversuch zur Demonstration geringer Löslichkeit in der Wärme empfehlen.

Die Formel des Salzes ist $\mathrm{Ce}_{2}\left(\mathrm{SO}_{3}\right)_{3}+9 \mathrm{H}_{2} \mathrm{O}$. JoLIN ${ }^{1}$ erhielt eim Salz von der Formel $\mathrm{Ce}_{2}\left(\mathrm{SO}_{3}\right)_{3} \cdot 3 \mathrm{H}_{2} \mathrm{O}$.

$$
\begin{aligned}
& 0.2700 \mathrm{~g} \mathrm{~S} \text { ergaben } 0.1370 \mathrm{~g} \mathrm{CeO}_{2} \\
& 0.3040 \mathrm{~g} \mathrm{~S} \text { ergaben } 0.1550 \mathrm{~g} \mathrm{CeO}_{2} \\
& 0.2950 \mathrm{~g} \mathrm{~S} \text { ergaben } 0.3000 \mathrm{~g} \mathrm{BaSO}_{4} .
\end{aligned}
$$

Ber. Ce 41.06; S 14.10 .

Gef. Ce $41.30,41.50 ;$ S 13.97 .

Die Sulfite des Praseodyms und Neodyms dürften dem Cersulfit sich ähnlich verhalten, wie die Untersuchung des Didymsulfits durch MARIGNAC ${ }^{2}$ zeigte.

\section{Zusammenfassung der Hauptergebnisse:}

I. Neutrales Natriumsulfit fällt die dreiwertigen Ceriterden quantitativ als neutrale Sulfite, während Thorium und Zirkoniumsalze im Überschufs des Fällungsmittels leicht lösliche, gallertartige Niederschläge basischer Sulfite liefern. In der Lösung des Thoriumsalzes ist die Existenz komplexer Alkalithoriumsulfite anzunehmen; in festem Zustand wurde das $\mathrm{Salz} \mathrm{Th}_{2}(\mathrm{OH})_{2}\left(\mathrm{SO}_{3}\right)_{3} \cdot 2 \mathrm{Na}_{2} \mathrm{SO}_{3}+\mathrm{aq}$ isoliert. Aus der Lösung in Kaliumsulfit liefs sich die Verbindung $\mathrm{Th}(\mathrm{OH})_{2} \mathrm{SO}_{3} \cdot 2 \mathrm{~K}_{2} \mathrm{SO}_{3}+\mathrm{aq}$ isolieren.

II. Die Trennungsmethode von Chavastelon ist bei geringem Thoriumgehalt, wie ihn der Monazitsand zeigt, praktisch unbrauchbar.

III. Neutrales Ammoniumsulfit fällt verdünnte Lösungen von Thoriumnitrat als basisches Sulfit, Zirkonnitrat gibt mit überschüssigem Sulfit eine klare Lösung, aus der sich nach 12 Stunden gallertartiges Ammoniumzirkonsulfit abscheidet.

1 Bull. soc. chim. [2] 21, 539.

2. 1 . e. 
IV. Die Tendenz zar Bildung von Alkalidoppelsulfiten ist bei den stărker basischen Ceriterden nicht vorkanden. Die Löslichkeit der Hydroxyde in wässeriger $\mathrm{SO}_{2}$ nimmt in folgender Reihenfolge ab: Ce, Di, La, Th. Die Sulfite fallen als nentrale Salze aus den Lösungen bei Gegenwart freier Süure beim Erwărmen aus.

Herrn Privatdozent Dr. R. J. Mryer möchte ich für die Überlassung einer Reihe wertroller Präparate und seinen mir jederzeit frenndlichst gewährten Rat meinen besten Dank aussprechen.

Berlin N, Wissenschaftlich-chemisohes Institut.

Bei der Redaktion eingegangen am 20. Februar 1905. 